\title{
What do we know about eye movements in Alzheimer's disease? The past 37 years and future directions
}

\section{"...our exact knowledge of eye movements in Alzheimers disease for the last four decades is due to one study."}

First draft submitted: 1 April 2016; Accepted for publication: 4 April 2016; Published online: 27 June 2016

Keywords: Alzheimer's disease $\bullet$ eye movements $\bullet$ review

\section{Introduction}

The goal of this Biomarkers in Medicine Editorial is to exhaustively describe our current knowledge of eye movements in Alzheimer's disease (AD) in the field of neurology. To achieve this goal, 97 publications from the MedLine $^{\circledR}$ database were examined as a result of crossed 'Alzheimer disease' and 'eye movements' subheadings. I discarded 12 review articles (for recent and complete panoramas see $[1,2]$ ) and 12 other articles, which were insufficiently relevant to the subject (due to weak bibliometric indexing). Seven further articles were also dismissed as they focused on atypical subsets of AD (such as amnestic or not mild cognitive impairment, Balint syndrome, posterior cerebral atrophy). This literature restriction led to a set of 66 relevant publications (our 100\% standard). It is noteworthy that 64 and 2 publications respectively concerned presumed (97.0\%) and confirmed (3.0\%) $\mathrm{AD}$, giving us the measure of the progress to be made. Among the articles in presumed AD, 27 studies $(40.9 \%)$ were performed in the field of oculomotor research, that is, eye movements were analyzed per se in order to determine eye movement indicators of $\mathrm{AD}$ presence or severity. In the remaining 37 studies $(56.1 \%)$, eye movements were used to explore the different cognitive functions that may be impaired in AD from low-level vision to social cognition. I will present the results in $\mathrm{AD}$ as compared to healthy partic- ipants (throughout the text), before suggesting future directions for basic and clinical research and summarizing our knowledge of eye movement and cognitive markers of $\mathrm{AD}$ diagnosis and prognosis.

\section{Eye movement research in presumed AD}

Oculomotor research has revealed different types of eye movement deficits, which may either early detect AD or correlate with its severity. Seminal study by Hutton et al. [3] reported three types of oculomotor disturbances during spontaneous visual exploration: 'poorly regulated gaze patterns', 'programming type of gaze perseveration' and 'staring gaze pattern'. Consistently, quantitative analysis of exploratory eye movements was suggested to early detect ADtype dementia [4]. During fixation, higher occurrence of saccadic intrusions was suggested to be sensitive to the progression of AD. Increased blink occurrence during fixation and pursuit, eye blink conditioning deficit [5], eye-head and eye-hand coordination impairments have also been reported in presumed AD. The literature on pursuit eye movements $(10.6 \%)$ has led to consensual findings. Pursuit in AD-type dementia has reduced gain and velocity and exhibits more catch-up saccades or saccadic intrusions, which are better defined as anticipatory saccades with higher amplitude [6]. Some pursuit indicators were reported to correlate

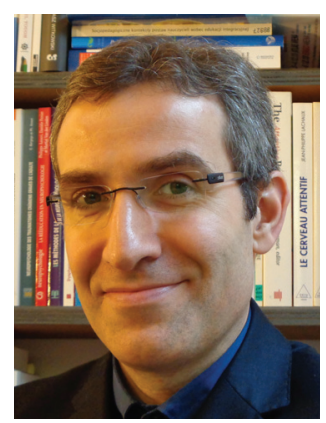

Olivier A Coubard The Neuropsychological Laboratory, CNS-Fed, 14 rue du Regard, 75006 Paris, France olivier.coubard@cns-fed.com 
with AD severity. Another $10.6 \%$ of literature was dedicated to saccades, with contradictory results. Pioneer research showed increased latency (i.e., reaction time) of reflexive saccades, which was correlated [7] or not [8] with dementia severity. Five studies examining reflexive prosaccades and voluntary antisaccades (e.g., [9-11]) corroborated increased latency of the former (except [10]) and additionally evidenced more systematic deficit in the latter. Indeed antisaccade measures appeared to be the most specific to AD. However, eye movements have not shown any superiority to neuropsychological testing to differentiate $\mathrm{AD}$ from other dementias.

“ ...27 studies ( $40.9 \%)$ were performed in the field of oculomotor research ... In the remaining 37 studies ( $56.1 \%$ ), eye movements were used to explore the different cognitive functions..."

\section{Eye movements to explore cognition in presumed AD}

Exploratory eye movement disturbances are only one aspect of vision deficits in AD-type dementia including altered acuity, contrast sensitivity, visual field, color and motion perception, visual categorization and EEG abnormalities. Eye movements are also a 'window' to explore the dysfunctional brain in AD. Performance in visually-guided saccades worsens with increasing number of spatial locations to attend, while fixation and gazing parameters indicate reduced visuospatial ability in clock reading and impaired visuoconstructional skill in copying and drawing. Consistently an fMRI study evidenced reduction in right intraparietal sulcus activation suggesting that left-dominant laterality ratio for intraparietal sulcus may be a marker of AD progression [12]. The study of selective attention has revealed an excess of saccades, fixation and pupil dilation in visual search and feature-and-conjunction search, which is interpreted as dynamic adjustment of attentional zoom or loss of access to attentional resources, consistent with compensatory over recruitment of frontoparietal attention network [13]. An important set of literature (12.1\%) was devoted to one attentional control process - inhibition. Presumed AD exhibits higher reflexive error occurrence in NoGo, Go/NoGo and antisaccade tasks [14], distractibility to moving stimuli [15], but relatively preserved inhibition of return, prediction in pursuit, antisaccade gap/simultaneous/overlap effects. Antisaccade measures [14] and errors in line bisection with background movement [15] were reported to correlate with dementia severity. Reading is also impaired in presumed $\mathrm{AD}$ : slowness, more saccade regressions, less words with one fixation, shorter forward saccades and the absence of contextual word predictability effect [16].

\section{Eye movements in autopsy-confirmed AD}

Importantly, our exact knowledge of eye movements in $\mathrm{AD}$ for the last four decades is due to one study. Boxer $e t$ al. [17] invited ten participants with autopsyconfirmed AD, 27 with autopsy-confirmed frontotemporal dementia (FTD, including 12 frontotemporal lobar degeneration associated with insoluble deposits of the TAR DNA-binding protein 43, FTLD-TDP; eight progressive supranuclear palsy, PSP; four corticobasal degeneration; three Pick disease or FTD and parkinsonism linked to chromosome 17) to perform 35.6 months prior to death 28 reflexive horizontal or vertical saccades (seven left, right, up and down) and 36 horizontal antisaccades (18 left and right), as compared to 27 healthy controls (HC). Analyses (of variance with Clinical Dementia Rating-Sum of Boxes as covariate and Tukey/Sidak post hoc statistics) revealed that $\mathrm{AD}$ causes increased horizontal saccade latency as compared to FTLD-TDP and PSP, increased vertical saccade latency as compared to FTLD-TDP and $\mathrm{HC}$, and lower rate of correct antisaccades than HC considering or not self-correction. Diagnostic value of saccade parameters from receiver operating curve analysis unveiled that only horizontal saccade latency differentiates AD from all FTDs. Another study [18] evidenced in 30 autopsy cases that AD-related cytoskeletal changes in the rostral interstitial nucleus of the medial longitudinal fascicle are slight, pronounced and severe in respectively cortical neurofibrillary tangle/neuropil thread stages I-II, III-IV and V-VI, corresponding respectively to preclinical, incipient and clinical phases of $\mathrm{AD}$, while $\beta$-amyloid deposits only appear in its final stage, suggesting that vertical saccade slowing might be a marker of $\mathrm{AD}$ progression.

\section{Future research}

Directions for future research are six-fold. First, basic oculomotor research may study unexplored eye movements in AD such as optokinetic nystagmus, fixational tremor and drifts or vergence. Second, basic psychology may develop eye movement paradigms to examine cognitive functions which decline in AD: consciousness, metacognition, self-movement, self-defining memories, etc. Third, there is a need for more interaction between clinical neurologists and basic scientists to refresh what is crucial to study. At early phases of $\mathrm{AD}$ before attention and memory deficits take place insidious apathy and divestment measurable at motor, cognitive and social levels. Three studies by the same team have revealed reduced curiosity as measured by lower reaction to novelty and incongruity [19]. AD 
participants fixate less on faces and are impaired in facial expression recognition though they keep mutual gaze and prefer to fixate or pursue faces, enough to inhibit vestibulo-ocular reflex [20]. Fourth, research is lacking creativity once the different types of movements and cognitive functions have been examined. Time has come to inject theory into neurology, combining mathematics and physiology, to test predictions about underlying physiological mechanisms and their decline in AD. Fifth, studies should be conducted in autopsy-confirmed AD or at least in homogenous cohorts of patients based on genetic, imaging and neuropsychological data. Sixth, future research belongs to multidimensional analyses to serve diagnosis and prognosis purposes. Eye movements may be precious to detect $\mathrm{AD}$ and quantify interventions based on pharmacology, physical and cognitive training, and prevention of dementia-induced anxiety, social isolation and suicide/homicide.

\section{Eye movement \& cognitive markers in AD}

I summarize here eye movement and cognitive markers in $\mathrm{AD}$.

\section{Eye movement \& cognitive markers in autopsy-confirmed AD}

- Diagnosis:

- Reflexive horizontal predictable-in-time saccades: increased latency [17];

- Reflexive vertical predictable-in-time saccades: increased latency [17];

= Antisaccades: lower antisaccade rate, lack of self-correction [17].

- Severity/progression:

= Reflexive vertical saccades: reduced velocity? [18].

\section{Eye movement \& cognitive markers in presumed $A D$}

- Diagnosis:

- Spontaneous eye movements: quantitative analysis [4];

- Blinks: higher occurrence during fixation/ pursuit; eye blink conditioning deficit [5];

- Reflexive saccades: increased latency;

- Reflexive unpredictable-in-time saccades: reduced gain, higher anticipatory saccade occurrence;
- Antisaccades: lower antisaccade rate, increased antisaccade latency, higher reflexive error rate, lack of self-correction;

- Reflexive saccades and antisaccades: higher latency variability, preserved gap/simultaneous/ overlap effects;

= Reading: higher saccade regression occurrence, lower one-fixation word occurrence, reduced forward saccade amplitude, lack of contextual word predictability effect [16]?;

- Curiosity: reduced reaction to novelty/incongruity, normalized by emotional arousal [19]?;

- Social cognition: facial expression deficit, preserved mutual gaze and preference to look at faces?;

- Severity/progression:

- Fixation: higher saccadic intrusion occurrence;

- Pursuit: reduced gain, reduced velocity, increased catch-up saccade/saccadic intrusion/anticipatory saccade occurrence, increased anticipatory saccade amplitude;

$=$ Reflexive saccades: increased latency [7,8]?;

- Antisaccades: higher reflexive error rate;

- Visuospatial: fMRI left-dominant laterality ratio for intraparietal sulcus [12];

- Visuospatial: error rate in line bisection with background movement [15];

- Reading: higher saccade regression occurrence, lower one-fixation word occurrence, reduced forward saccade amplitude [16]?

\section{Acknowledgements}

The author thanks Biomarkers in Medicine editors for their invitation to submit this editorial.

\section{Financial \& competing interests disclosure}

The author has no relevant affiliations or financial involvement with any organization or entity with a financial interest in or financial conflict with the subject matter or materials discussed in the manuscript. This includes employment, consultancies, honoraria, stock ownership or options, expert testimony, grants or patents received or pending, or royalties.

No writing assistance was utilized in the production of this manuscript. 


\section{References}

1 Przedpelska-Ober E. Zaburzenia ruchów galek ocznych w chorobie Alzheimera [Eye movements dysfunctions in Alzheimer's disease]. Neurol. Neurochir. Pol. 40(1), 34-41 (2006).

2 Molitor RJ, Ko PC, Ally BA. Eye movements in Alzheimer's disease. J. Alzheimers Dis. 44(1), 1-12 (2015).

3 Hutton JT, Johnston CW, Shapiro I, Pirozzolo FJ. Oculomotor programming disturbances in the dementia syndrome. Percept. Mot. Skills 49(1), 312-314 (1979).

4 Nakashima Y, Morita K, Ishii Y, Shouji Y, Uchimura $\mathrm{N}$. Characteristics of exploratory eye movements in elderly people: possibility of early diagnosis of dementia. Psychogeriatrics 10(3), 124-130 (2010).

5 Woodruff-Pak DS, Papka M, Romano S, Li YT. Eyeblink classical conditioning in Alzheimer's disease and cerebrovascular dementia. Neurobiol. Aging 17(4), 505-512 (1996).

6 Zaccara G, Gangemi PF, Muscas GC et al. Smooth-pursuit eye movements: alterations in Alzheimer's disease. J. Neurol. Sci. 112(1), 81-89 (1992).

7 Pirozzolo FJ, Hansch EC. Oculomotor reaction time in dementia reflects degree of cerebral dysfunction. Science 214(4518), 349-351 (1981).

8 Hershey LA, Whicker L Jr, Abel LA, Dell'osso LF, Traccis S, Grossniklaus D. Saccadic latency measurements in dementia. Arch. Neurol. 40(9), 592-593 (1983).

9 Shafiq-Antonacci R, Maruff P, Masters C, Currie J. Spectrum of saccade system function in Alzheimer disease. Arch. Neurol. 60(9), 1272-1278 (2003).

10 Mosimann UP, Müri RM, Burn DJ, Felblinger J, O’Brien JT, Mckeith IG. Saccadic eye movement changes in Parkinson's disease dementia and dementia with Lewy bodies. Brain 128(Pt 6), 1267-1276 (2005).

11 Garbutt S, Matlin A, Hellmuth J et al. Oculomotor function in frontotemporal lobar degeneration, related disorders and Alzheimer's disease. Brain 131(Pt 5), 1268-1281 (2008).
12 Thulborn KR, Martin C, Voyvodic JT. Functional MR imaging using a visually guided saccade paradigm for comparing activation patterns in patients with probable Alzheimer's disease and in cognitively able elderly volunteers. AJNR Am. J. Neuroradiol. 21(3), 524-531 (2000).

13 Viskontas IV, Boxer AL, Fesenko J et al. Visual search patterns in semantic dementia show paradoxical facilitation of binding processes. Neuropsychologia 49(3), 468-478 (2011).

14 Crawford TJ, Higham S, Renvoize T et al. Inhibitory control of saccadic eye movements and cognitive impairment in Alzheimer's disease. Biol. Psychiatry 57(9), 1052-1060 (2005).

15 Kim EJ, Lee BH, Seo SW et al. Attentional distractibility by optokinetic stimulation in Alzheimer disease. Neurology 69(11), 1105-1112 (2007).

16 Fernández G, Schumacher M, Castro L, Orozco D, Agamennoni O. Patients with mild Alzheimer's disease produced shorter outgoing saccades when reading sentences. Psychiatry Res. 229(1), 470-478 (2015).

17 Boxer AL, Garbutt S, Seeley WW et al. Saccade abnormalities in autopsy-confirmed frontotemporal lobar degeneration and Alzheimer disease. Arch. Neurol. 69(4), 509-517 (2012).

18 Rüb U, Del Tredici K, Schultz C, Büttner-Ennever JA, Braak $\mathrm{H}$. The premotor region essential for rapid vertical eye movements shows early involvement in Alzheimer's diseaserelated cytoskeletal pathology. Vision Res. 41(16), 2149-2156 (2001).

19 Labar KS, Mesulam M, Gitelman DR, Weintraub S. Emotional curiosity: modulation of visuospatial attention by arousal is preserved in aging and early-stage Alzheimer's disease. Neuropsychologia 38(13), 1734-1740 (2000).

20 Riestra AR, Heilman KM. Visual facial grasp. Neurocase 10(5), 363-365 (2004). 